\title{
Hierarchical Precoding in a Realistic Ultradense Heterogeneous Environment Exceeding the Degrees of Freedom
}

\author{
Mohamed Shehata, ${ }^{1}$ Martin Kurras, ${ }^{2}$ Khaled Hassan, ${ }^{3}$ and Lars Thiele ${ }^{2}$ \\ ${ }^{1}$ Information Engineering and Technology, German University in Cairo, Cairo, Egypt \\ ${ }^{2}$ Fraunhofer Heinrich Hertz Institute, Berlin, Germany \\ ${ }^{3}$ Fraunhofer Institute for Integrated Circuits (IIS), Erlangen, Germany \\ Correspondence should be addressed to Martin Kurras; martin.kurras@hhi.fraunhofer.de
}

Received 19 February 2016; Revised 14 July 2016; Accepted 21 July 2016

Academic Editor: Giuseppe Castaldi

\begin{abstract}
Copyright (C) 2016 Mohamed Shehata et al. This is an open access article distributed under the Creative Commons Attribution License, which permits unrestricted use, distribution, and reproduction in any medium, provided the original work is properly cited.
\end{abstract}

Cell densification is a widely used approach to increase the spectral efficiency per area of cellular networks. Such Ultradense Networks (UDNs) consisting of small cells are often coordinated by macro base stations (BSs). With universal frequency reuse interference from the macro BS limits the system spectral efficiency. In this work we exploit the degrees of freedom at the macro BS to apply interference coordination. We propose a hierarchical precoding strategy in the spatial domain in order to project interference from the macro BS into the subspace of small cell users enabling linear cancellation. The macro BS interference towards small cell users is aligned within the joint null space of users served by the macro BS. Compared to classical interference alignment, our scheme does not require coordination between macrocells and small cells. We present three algorithms: in the first the interference is minimized by iterative alignment, in the second the uncoordinated interference from the small cells is considered, and in the third iterative Minimum Mean Square Error (MMSE) technique is used. We provide numerical evaluation, complexity analysis, and robustness analysis of these algorithms based on a realistic channel model showing the benefit of hierarchical precoding compared to the uncoordinated case.

\section{Introduction}

Throughout the last decades, wireless communication technologies witnessed a large number of challenges such as the great evolution in data traffic and the massive increase in the amount of mobile User Equipment (UE). Thus, one of the main objectives for future wireless technologies is supplying the UEs with high data rates in order to support real time applications. Another objective is extending the coverage and supporting more UEs, while taking into account the scarcity of the current licensed spectrum resources.

This continuous increase in the data traffic and number of UEs can be satisfied by using a combination of more spectrum resources, enhancing spectrum efficiency [1], and cell densification. One approach to achieve this combination is Ultradense Network (UDN) deployment. Such dense deployments are often in the form of a heterogeneous network (HetNet) [2-4], where many small cells are deployed having less capabilities, that is, less transmit power than the macro base stations (BSs); see Figure 1. In a universal frequency reuse, this power imbalance results in interference limitation at users served by these small cells. This interference limitation is caused by the macro BS transmitting to users which are not in the coverage of a small cell. Therefore, the focus in this paper is on increasing the system spectral efficiency by spatial interference coordination from the macro BS to small cell users in a HetNet. The idea is to utilize the degrees of freedom at the macro BSs from the "large" number of antennas. Compared to massive Multiple-Input MultipleOutput (MIMO) [5, 6] originated in [7] where the number of antennas is assumed to be infinity, we focus on 4 and 8 antennas, already provided in the standard [8]. The degrees of freedom are used to project interference from the macro BS towards small cell connected UEs such that it can be canceled with linear receivers. Simultaneously the macro BS still serves UEs which are not in the coverage of a small cell. 
Hence, we propose a two-stage hierarchical precoding/beamforming approach to protect the small cell connected UEs from the macrointerference, while serving the macro UEs simultaneously. This is achieved by combining the idea of interference alignment (IA) $[9,10]$ by iterative minimization [11] with operating in the null space of macro UEs served by Block Diagonalization (BD) [12] in a twostage or hierarchical, precoding approach. This is achieved by modifying an iterative IA algorithm introduced in [11, $13,14]$ to operate in the joint null space of the macro UEs obtained using BD. Thus, we ensure interference decoupling of macro and small cell connected UEs. In [15] we presented two extensions of the algorithm presented in [14]. In the first algorithm called "Block Diagonalization-Interference Alignment (BDIA) uncoordinated" uncoordinated interference from small cells or surrounding macro BSs is considered. In the second algorithm, the iterative IA objective is changed to Minimum Mean Square Error (MMSE), called "BDIA MMSE Joint Transmit and Receive (JTR) structure.”

These algorithms require that Channel State Information (CSI) at the transmitter (CSIT) from the macro and small cell connected UEs is available. As a baseline we assume having perfect knowledge of the CSI and then we extend our work to include the imperfect CSI scenario. We also extend our work in [15] by evaluating the spatial hierarchical precoding schemes in a more realistic ultradense HetNet. Thus, we exceed the macro BS degrees of freedom with up to 10 small cells in the coverage area of a macro BS. Moreover, we consider the out-of-cell interference from other macro BSs tiers in the hierarchical precoding framework. This can be achieved by utilizing the advantage of adding the MMSE pre- and postcoder to the proposed hierarchical framework. Finally, we provide a comprehensive study on the contradicting effects of the proposed hierarchical algorithms on both the signal and interference received power, as well as spectral efficiency separately for macro and small cell attached UEs.

The novel contributions in this work are summarized as follows.

(1) A complexity analysis and comparison of the algorithms are presented. In this analysis we separate the required steps of the algorithms and provide the complexity required in each of the steps.

(2) Furthermore, we investigated the trade-off on the system spectral efficiency exceeding the degrees of freedom available at the macro BS. In an UDN the number of small cells can easily exceed the number of antennas at the macro BS.

(3) Finally, we analyze the "robustness" of our proposed algorithms. In [11, 13-16] perfect channel knowledge is considered, which is why we introduce a channel uncertainty and investigate in this work its impact on the system spectral efficiency. Additionally, we also take into account the impact from multiple macro BSs and show the advantage of our proposed algorithms increasing the number of antennas and therefore degrees of freedom at the macro BS.

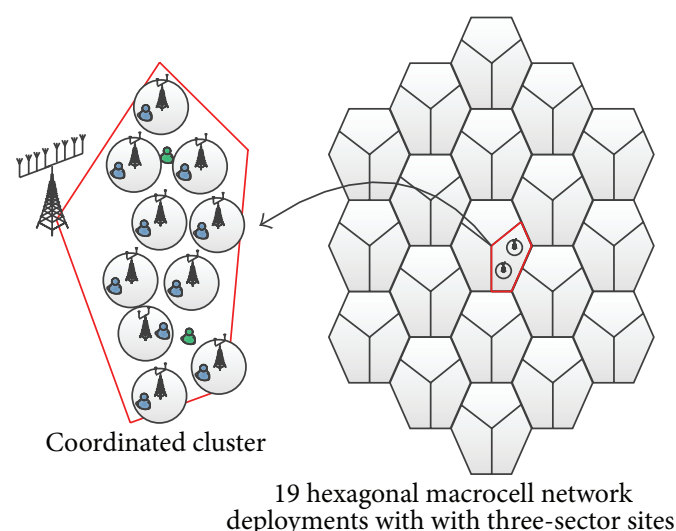

$\begin{array}{ll}8 \text { Macrouser } & \text { Sector of interest } \\ 8 \text { Picouser } & \text { (3) Picocell }\end{array}$

FIGURE 1: Deployment and transmission mode of the heterogeneous network.

Remark 1. Throughout our work, all the processing for the hierarchical precoding scheme is handled at the macro BS, which enables independent transmission at the small cells. This implies that no user data or feedback exchange is required between transmit nodes.

For notational convenience, throughout the whole paper, a scalar is denoted as $x$, a vector as $\mathbf{x}$, and a matrix as $\mathbf{X}$. Matrix conjugate transpose is denoted as $(\cdot)^{H}$. The most dominant eigenvector of a matrix $(\cdot)$ is expressed as $\xi_{\max }(\cdot)$, which is the eigenvector corresponding to the largest eigenvalue, while the least dominant one is expressed as $\xi_{\min }(\cdot)$. The Frobenius norm is represented as $\|(\cdot)\|_{F}^{2}$. The matrix $\mathbf{I}_{N}$ denotes $N \times N$ identity matrix. $\mathbb{E}\{\cdot\}$ stands for the expectation operator.

\section{System Model}

In this work we consider the HetNet deployment shown in Figure 1 and investigate the downlink scenario within coherent channel bandwidth. The system considered is a Multiuser (MU-) MIMO Orthogonal Frequency Division Multiplexing (OFDM) system with constant CSI per Resource Block (RB). The deployment is a central macro BS serving a specified set of UEs within its transmission range and surrounded by multiple tiers of macrosites. Each macrosite is divided into three $120^{\circ}$ sectors and within each sector small cells are deployed. Each macrosector and each small cell has an independent cell identity (ID) such that we have a total set of cells $\mathscr{M}$ with cardinality $M=|\mathscr{M}|$. We can split the set $\mathscr{M}$ into the disjoint subsets $\mathscr{M}_{r} \subset \mathscr{M}_{\text {and }} \mathscr{M}_{p} \subset \mathscr{M}_{\text {representing }}$ macrocells and small cells within the system, respectively, such that $\mathscr{M}_{r} \cup \mathscr{M}_{p}=\mathscr{M}_{\text {and }} \mathscr{M}_{r} \cap \mathscr{M}_{p}=\emptyset$.

Further, we assume that a macrosector together with the underlying small cells forms a cluster given by $\mathscr{M}_{c} \subset \mathscr{M}_{\text {with }}$ cardinality $M_{c}=\left|\mathscr{M}_{c}\right|$, for example, Figure 1 . Coordination between the macrocell and small cells is only done within the same cluster. Since we are assuming full spectral frequency reuse within $\mathscr{M}$, other macrosectors (and small cells) cause 
residual uncoordinated interference, henceforth, referred to as intercluster interference (ICI).

The set $\mathscr{K}$ represents all UEs located within the coverage area of a cluster (connected to a cell within the cluster) and $\mathscr{K}_{m} \subset \mathscr{K}$ a subset of UEs connected to $m$ th BS. Consequently the subset $\mathscr{K}_{r} \subset \mathscr{K}$ represents UEs connected to a macrocell and $\mathscr{K}_{p} \subset \mathscr{K}$ are the users attached to the small cells. Furthermore, we denote with $\mathscr{K}_{c} \subset \mathscr{K}$ the UEs selected (scheduled) for simultaneously spatial downlink transmission on the same time-frequency resource in cluster $\mathscr{M}_{c}$. The number of antennas at macrocells, small cells, and UEs is set to $N_{t}^{m}, N_{t}^{p}$, and $N_{r}$, respectively.

With these assumptions the received signal of user $k \epsilon$ $\mathscr{K}$ connected to cell $m$ on a time-frequency resource (RB) is defined as follows:

$$
\begin{aligned}
& \mathbf{y}_{k}=\underbrace{\mathbf{H}_{m, k} \mathbf{b}_{m, k} \sqrt{P_{m, k}}}_{\widetilde{\mathbf{L}}_{k}} \mathbf{x}_{k}+\underbrace{\sum_{j \in\left\{\mathscr{K}_{m} \backslash k\right\}} \mathbf{H}_{m, k} \mathbf{s}_{m, j}}_{\widetilde{\mathbf{s}}_{k}} \\
& +\underbrace{\sum_{n \in\left\{\mathscr{M}_{c} \backslash\{m\}\right\}} \sum_{\left.j \mathscr{K}_{n}\right\}} \mathbf{H}_{n, k} \mathbf{s}_{n, j}}_{\widetilde{\mathbf{T}}_{k}} \\
& +\underbrace{\sum_{l \in\left\{\mathscr{M} \backslash \mathscr{M}_{c}\right\}} \sum_{j \in\left\{\mathscr{K} \backslash \mathscr{K}_{l}\right\}} \mathbf{H}_{l, k} \mathbf{s}_{l, j}+\mathbf{n},}_{\widetilde{\mathbf{Z}}_{k}}
\end{aligned}
$$

where $\mathbf{H}_{l, u} \in \mathbb{C}^{N_{r} \times N_{t}^{l}}$ is the channel matrix between $l$ th BS and $u$ th UE. The vector $\mathbf{s}_{l, u}=\mathbf{b}_{l, u} \sqrt{p_{l, u}} \mathbf{x}_{u} \in \mathbb{C}^{N_{t}^{l} \times 1}$ denotes the precoded signal $\mathbf{x}_{u}$ with the precoding vector $\mathbf{b}_{l, u}$ and transmit power $p_{l, u} \leq P_{s}$ limited by a sum power constraint $P_{s}$. The vector $\mathbf{n}$ represents $\mathbb{C}^{N_{r} \times 1}$ Additive White Circular Symmetric Complex Gaussian Noise (AWCSCGN) samples at the receiver with covariance $\mathbb{E}\left\{\mathbf{n n}^{H}\right\}=\sigma^{2}{ }_{n} \mathbf{I}_{N_{r}}$. For clarity we clustered the receive signal of user $k$ into the 4 following parts: (1) $\widetilde{\mathbf{L}}_{k}$ the effective channel for the desired UE $k$, (2) $\widetilde{\mathbf{S}}_{k}$ interstream (or multiuser) interference caused by spatially multiplexed users at the serving cell $m$, (3) $\widetilde{\mathbf{T}}_{k}$ intracluster interference which is caused by other cells within the same cluster $\mathscr{M}_{c} \backslash m$, and (4) $\widetilde{\mathbf{Z}}_{k}$ intercluster interference from the surrounding tiers $\mathscr{M} \backslash \mathscr{M}_{c}$ in addition to thermal noise.

The resulting Signal to Interference and Noise Ratio (SINR) of user $k$ used to obtain the spectral efficiency with Shannon's formula is thus given as follows:

$$
\operatorname{SINR}_{k}=\frac{\left|\mathbf{w}_{k}^{H} \mathbf{H}_{m, k} \mathbf{b}_{m, k} \sqrt{p_{m, k}}\right|^{2}}{\underbrace{\left|\mathbf{w}_{k}^{H} \widetilde{\mathbf{S}}_{k}\right|^{2}}_{\text {Inter Stream IF }}+\underbrace{\left|\mathbf{w}_{k}^{H} \widetilde{\mathbf{T}}_{k}\right|^{2}}_{\text {Intra Cluster IF }}+\underbrace{\left|\mathbf{w}_{k}^{H} \widetilde{\mathbf{Z}}_{k}\right|^{2}}_{\text {Inter Cluster IF }}},
$$

where $\mathbf{w}_{k}$ is the postcoder (receive beamformer) at $k$ th UE. Using optimal linear equalization the MMSE postcoder $\mathbf{w}_{k}$ is given by

$$
\mathbf{w}_{k}=\left(\widehat{\mathbf{Q}}_{k}\right)^{-1} \widetilde{\mathbf{L}}_{k}
$$

where the covariance matrix $\mathbf{Q}_{k}$ comprises

$$
\widehat{\mathbf{Q}}_{k}=\widetilde{\mathbf{S}}_{k} \widetilde{\mathbf{S}}_{k}^{H}+\widetilde{\mathbf{T}}_{k} \widetilde{\mathbf{T}}_{k}^{H}+\widetilde{\mathbf{Z}}_{k} \widetilde{\mathbf{Z}}_{k}^{H}+\widetilde{\mathbf{L}}_{k} \widetilde{\mathbf{L}}_{k}^{H} .
$$

In this paper we also inspect the case of imperfect CSI and we model the channel after adding the error as follows:

$$
\widehat{\mathbf{H}}=\sqrt{1-\varepsilon} \mathbf{H}+\sqrt{\varepsilon} \mathbf{H}_{e},
$$

where $\widehat{\mathbf{H}}$ represents the channel with error, while $\mathbf{H}$ represents the original channel, $\varepsilon$ is the channel error variance, and $\mathbf{H}_{e} \in$ $\mathbb{C}^{N_{r} \times N_{t}} \widetilde{N}(0, \sigma)$ represents the error added to the channel.

\section{Algorithms}

The basic concept in this paper is exceeding the macro degrees of freedom (DoF), by serving a number of UEs simultaneously within the cluster which is greater than the number of transmit antennas at the macro BS. Then we inspect the effect of this on the hierarchical precoding schemes presented in [15] and whether we can still achieve a gain over the uncoordinated case or not. In the uncoordinated case the macro BS only applies BD without taking into account the interference towards the underlying small cells. However, with applying the hierarchical precoding schemes at the macro BS, it can utilize the free spatial dimensions available to align its interference towards the underlying small cells. Here in this section we will give an introduction for the schemes with further discussion of the coordination mechanism between the macro BS, small cells, and the UEs within the cluster.

3.1. Macrointerference Subspace Reduction. This algorithm referred to as BDIA requires two stages of precoding in a hierarchical way. The first precoding stage is applying $\mathrm{BD}$ [12] in order to mitigate the interstream interference between the spatially multiplexed macro UEs. The second stage is applying iterative IA [11] precoding to reduce the rank of the macrointerference subspace towards the small cell connected UEs, while preserving the orthogonal streams towards the served macro UEs. Then the two-stage precoder can be calculated as follows:

$$
\mathbf{b}_{m, k}=\widetilde{\mathbf{V}}_{k}^{0} \mathbf{f}_{k}
$$

where $\widetilde{\mathbf{V}}_{k}^{0}$ is the common null space of the scheduled macro UEs $\mathscr{K}_{r}$ derived from the BD algorithm and $\mathbf{f}_{k}$ from the IA algorithm. This second part of (6) $\mathbf{f}_{k}$ effectively reduces the interference subspace from the macrocell towards the small cell connected UEs in the same cluster $\mathscr{M}_{c}$ to a single dimension, so our objective here can be modelled as minimizing the interference leakage from the macro BS towards the small cell connected UEs as follows:

$$
\min \sum_{j \in \mathscr{K}_{n}} \sum_{k \in \mathscr{K}_{c}, k \neq j}\left\|\widehat{\mathbf{H}}_{j, k} \mathbf{f}_{k}-\mathbf{c}_{k} \mathbf{c}_{k}^{H} \widehat{\mathbf{H}}_{j, k} \mathbf{f}_{k}\right\|_{F}^{2},
$$

where $\mathscr{K}_{n} \in\left\{\mathscr{K}_{c} \backslash \mathscr{K}_{p}\right\}$ represents the macro UEs connected to BS $n$ within the cluster and $\mathbf{c}_{k}$ is rank 1 orthonormal basis for the received interference subspace $\boldsymbol{\Omega}_{k}$ and is calculated as follows:

$$
\mathbf{c}_{k} \cong \xi_{\max }\left(\sum_{j \in \mathscr{K _ { n }}, j \neq k} \widehat{\mathbf{H}}_{j, k} \mathbf{f}_{j} \mathbf{f}_{j}^{H} \widehat{\mathbf{H}}_{j, k}^{H}\right),
$$


while $\widehat{\mathbf{H}}_{j, k}$ represents the effective channels, such that the transmitting macro BSs are considered as $\mathscr{K}_{n}$ transmitters causing interference towards the small cell connected UEs and these new virtual effective channels can be calculated as

$$
\widehat{\mathbf{H}}_{j, k}=\mathbf{H}_{n, k} \widetilde{\mathbf{V}}_{j}^{0} \text {; }
$$

then the corresponding postcoder for each user $k \in \mathscr{K}_{c}$ is given as follows:

$$
\mathbf{w}_{k}=\mathbf{I}_{N_{r}}-\mathbf{c}_{k} \mathbf{c}_{k}^{H} .
$$

Then the IA precoder is calculated as

$$
\mathbf{f}_{j} \cong \xi_{\min }\left(\sum_{k \in \mathscr{K}_{c}, k \neq j} \widehat{\mathbf{H}}_{j, k}^{H} \mathbf{w}_{k} \widehat{\mathbf{H}}_{j, k}\right) \text {. }
$$

Then both the precoding filter at the macro BS and the interference subspace at the receivers of the UEs are updated iteratively until convergence. Here in Figure 2 the mechanism of the BDIA algorithm is shown.

Algorithm 2 (macrointerference subspace reduction).

(1) Initialize $\mathbf{f}_{j} \in C \forall j \in \mathscr{K}_{n}$ randomly such that $\mathbf{f}_{j} \mathbf{f}_{j}^{H}=$ 1.

(2) Calculate $\mathbf{f}_{s} \in C \forall s \in \mathscr{K}_{c} \backslash \mathscr{K}_{n}$ using BD [12].

(3) Calculate $\mathbf{c}_{k}$ for all $k \in \mathscr{K}_{c}$ using (8).

(4) Calculate $\mathbf{f}_{j}$ for all $j \in \mathscr{K}_{n}$ using (11).

(5) Repeat (3) and (4) until convergence.

3.2. Macrointerference Subspace Reduction with Considering Uncoordinated Interference. The "BDIA uncord." algorithm is similar to Algorithm 2, but it is more sophisticated because it takes into account the uncoordinated interference as mentioned in [13]. As uncoordinated interference we consider the undesired signal from the small cells towards the macro UEs and other small cells UEs in the case when only one cooperation area is active. When the multiple surrounding tiers are active also, then all the interference from BSs outside the cluster is added to the uncoordinated interference. Simply by replacing (8) by (12) in step (3) in Algorithm 2 this uncoordinated interference is taken into account in the hierarchical precoding framework. Henceforth, the orthonormal basis $\mathbf{c}_{k}$ for the received interference subspace is now calculated as the maximum eigenvector of the uncoordinated interference added to the interference subspace from the macro BS towards the small cell connected UEs. In this addition the macro BS transmitting power cannot be directly normalized as in (8) because of adding the uncoordinated interference to the macro interference leakage. This uncoordinated interference is arising from both the macrocells and the small cells, and each of them has different transmitting power. Thus, the orthonormal basis $\mathbf{c}_{k}$ for the received interference subspace is represented as follows:

$$
\mathbf{c}_{k} \cong \xi_{\max }(\sum_{j \in \mathscr{K}_{n}, j \neq k} \widehat{\mathbf{H}}_{j, k} \mathbf{f}_{j} p_{n} \mathbf{f}_{j}^{H} \widehat{\mathbf{H}}_{j, k}^{H}+\underbrace{\widetilde{\mathbf{P}}_{k}+\widetilde{\mathbf{R}}_{k}}_{\widetilde{\mathbf{Z}}_{k}}),
$$

where $\widetilde{\mathbf{P}}_{k}$ represents the uncoordinated interference from the small cells towards the macro UEs and other small cells UEs within the same cluster and is calculated as

$$
\widetilde{\mathbf{P}}_{k}=\sum_{r \in\left\{\mathscr{M}_{c} \backslash \mathscr{M}_{r}\right\}} \mathbf{H}_{r, k} \mathbf{f}_{r} p_{r} \mathbf{f}_{r}^{H} \mathbf{H}_{r, k}^{H}
$$

while $\widetilde{\mathbf{R}}_{k}$ represents the uncoordinated interference from the other BSs outside the cluster (intercluster interference) in case multicluster (MC) scenario is applied and is calculated as

$$
\widetilde{\mathbf{R}}_{k}=\sum_{l \in\left\{\mathscr{M} \backslash \mathscr{M}_{c}\right\}} \mathbf{H}_{l, k} \mathbf{f}_{l} p_{l} \mathbf{f}_{l}^{H} \mathbf{H}_{l, k}^{H} .
$$

Algorithm 3 (macrointerference subspace reduction with considering uncoordinated interference).

(1) Initialize $\mathbf{f}_{j} \in C \forall j \in \mathscr{K}_{n}$ randomly such that $\mathbf{f}_{j} \mathbf{f}_{j}^{H}=$ 1.

(2) Calculate $\mathbf{f}_{s} \in C \forall s \in \mathscr{K}_{c} \backslash \mathscr{K}_{n}$ using BD [12].

(3) Calculate $\mathbf{c}_{k}$ for all $k \in \mathscr{K}_{c}$ using (12).

(4) Calculate $\mathbf{f}_{j}$ for all $j \in \mathscr{K}_{n}$ using (11).

(5) Repeat (3) and (4) until convergence.

3.3. Macrointerference Subspace Reduction Using Iterative MMSE Transmit and Receive Structure. Here in this algorithm, referred to as BDIA MMSE JTR, the idea of iterative subspace refinement, where both the precoding filter at the macro BS and the interference subspace at the receivers of the UEs are updated iteratively, is utilized again, but this time using MMSE transmit and receive structures as in [13] with the target of taking the macro UE signal level into account, thus aligning the interference towards the small cells and at the same time keeping the macro UEs signal at a high level above the noise.

First the algorithm starts iterating in the forward direction by calculating the interference covariance matrix as follows:

$$
\mathbf{Q}_{k}=\sigma^{2} \mathbf{I}_{N_{r}}+\sum_{j \in \mathscr{K}_{n}, j \neq k} \widehat{\mathbf{H}}_{j, k} \mathbf{f}_{j} \mathbf{f}_{j}^{H} \widehat{\mathbf{H}}_{j, k}^{H}+\underbrace{\widehat{\mathbf{R}}_{k}+\widetilde{\mathbf{R}}_{k}}_{\widetilde{\mathbf{Z}}_{k}},
$$

where the effective channel here is given by $\widehat{\mathbf{H}}_{j, k}=$ $\mathbf{H}_{n, k} \widetilde{\mathbf{V}}_{j}^{0} \sqrt{p_{n, k}}$. With this the dominant interference subspace is obtained by

$$
\widehat{\mathbf{Q}}_{k} \cong \xi_{\max }\left(\mathbf{Q}_{k}\right)
$$

then calculating the signal subspace $\Phi_{k}$ as the whole receive space excluding the dominant interference subspace as in [11]:

$$
\boldsymbol{\Phi}_{k}=\mathbf{I}_{N_{r}}-\widehat{\mathbf{Q}}_{k} \widehat{\mathbf{Q}}_{k}^{H}
$$

The signal subspace is obtained in a different way in (17) in [13], where it is calculated as the minimum eigenvector of the interference subspace, but for consistency we stick throughout the whole work to the methodology in [11]. 


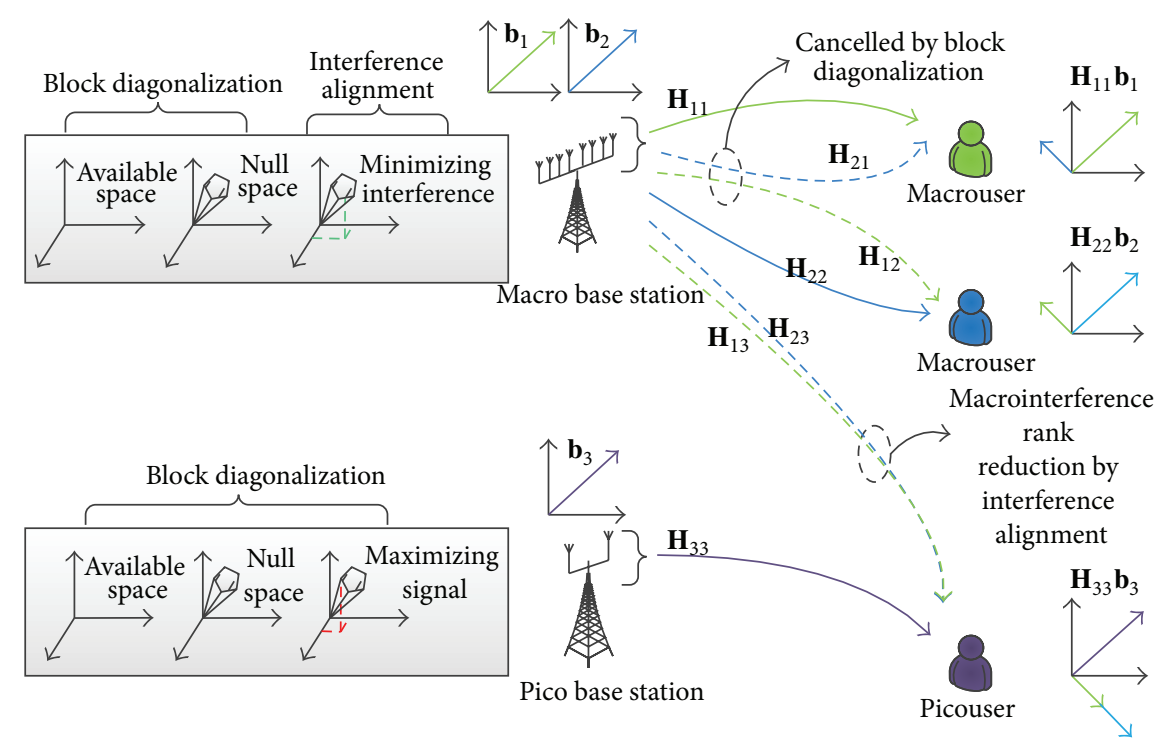

FIGURE 2: Macrointerference subspace reduction mechanism.

Then calculating the receive beamformer using the MMSE strategy for $\mathscr{K}_{c}$ cluster UEs where each macro UE is represented as $j \in \mathscr{K}_{n}$ and every small cell UE is given as $s \in\left\{\mathscr{K}_{c} \backslash \mathscr{K}_{n}\right\}$ and a small cell within the coordinated cluster is given as $p \in\left\{\mathscr{M}_{c} \backslash \mathscr{M}_{r}\right\}$, therefore the postcoder for the macro UEs is given as follows:

$$
\mathbf{w}_{j}=\left(\mathbf{Q}_{j}\right)^{-1} \widehat{\mathbf{H}}_{j, j} \mathbf{f}_{j},
$$

while for the small cell connected UEs is calculated as

$$
\mathbf{w}_{s}=\left(\mathbf{Q}_{s}\right)^{-1} \mathbf{H}_{p, s} \mathbf{f}_{s} \sqrt{p_{p, s}} .
$$

Then going backward step to calculate the interference covariance matrix $\overleftarrow{\mathbf{Q}}_{j}$,

$$
\overleftarrow{\mathbf{Q}}_{j}=\sigma^{2} \mathbf{I}_{\mathrm{DoF}_{j}}+\sum_{k \in \mathscr{K}_{c}, k \neq j} \widehat{\mathbf{H}}_{j, k}^{H} \mathbf{w}_{k} \mathbf{w}_{k}^{H} \widehat{\mathbf{H}}_{j, k},
$$

where $\mathrm{DoF}_{j}$ represents the available DoF at the macro BS for serving UE $j$ and is calculated as $\operatorname{DoF}_{j}=N_{T}-$ $\sum_{u \in \mathscr{K}_{c} \backslash \mathscr{K}_{p}, u \neq j} s_{u}$, where $s_{u}$ is the number of the spatial streams assigned for UE $u$; then the macro precoder $\mathbf{f}_{j}$ is calculated as follows:

$$
\mathbf{f}_{j}=\left(\overleftarrow{\mathbf{Q}}_{j}\right)^{-1} \widehat{\mathbf{H}}_{j, j}^{H} \mathbf{w}_{j} .
$$

Then the objective function is modelled as in (7):

$$
\min \sum_{j \in \mathscr{K}_{n} k \in \mathscr{K}_{c}, k \neq j}\left\|\widehat{\mathbf{H}}_{j, k} \mathbf{f}_{j}-\widehat{\mathbf{Q}}_{k} \widehat{\mathbf{Q}}_{k}^{H} \widehat{\mathbf{H}}_{j, k} \mathbf{f}_{j}\right\|_{F}^{2} .
$$

This algorithm is different from the one in Figure 2. The macro BS chooses the precoding vectors for its UE within the null space that minimizes the interference towards the small cell connected UEs, together with maximizing the signal level for the macro UEs.
Algorithm 4 (macrointerference subspace reduction using iterative MMSE transmit and receive structure).

(1) Initialize $\mathbf{f}_{j} \in C \forall j \in \mathscr{K}_{n}$ randomly such that $\mathbf{f}_{j} \mathbf{f}_{j}^{H}=$ 1.

(2) Calculate $\mathbf{f}_{s} \in C \forall s \in \mathscr{K}_{c} \backslash \mathscr{K}_{n}$ using BD [12].

(3) Calculate $\mathbf{Q}_{k}$ for all $k \in \mathscr{K}_{c}$ using (15).

(4) Calculate $\mathbf{w}_{j}$ for all $j \in \mathscr{K}_{n}$ using (18).

(5) Calculate $\mathbf{w}_{s}$ for all $s \in\left\{\mathscr{K}_{c} \backslash \mathscr{K}_{n}\right\}$ using (19).

(6) Backward step: calculate $\overleftarrow{\mathbf{Q}}_{j}$ for all $j \in \mathscr{K}_{n}$ using (20).

(7) Calculate $\mathbf{f}_{j}$ for all $j \in \mathscr{K}_{n}$ using (21).

(8) Repeat steps from (3) to (7) until convergence.

\section{Complexity Analysis}

In this section, we analyze the computational complexity of the presented IA based algorithms, including BDIA, BDIA uncord., and BDIA MMSE JTR, respectively.

First, we provide the reasons that show the importance of the computational complexity analysis of the proposed algorithms [17], which are

(1) IA based algorithms can exploit the channel reciprocity to calculate the transmit precoders and receiver beamformers in a distributed manner. Reciprocity is based on the Time Division Duplex (TDD) operation mode with synchronized time-slot. Practical wireless channel is time varying. Henceforth, assuming perfect reciprocity is not always accurate and can result in residual interference at the receiver side, thus, algorithms with low computational complexity (short computing time) are required to avoid performance loss arising from imperfect reciprocity in practical systems [18]. In our scenario we present 


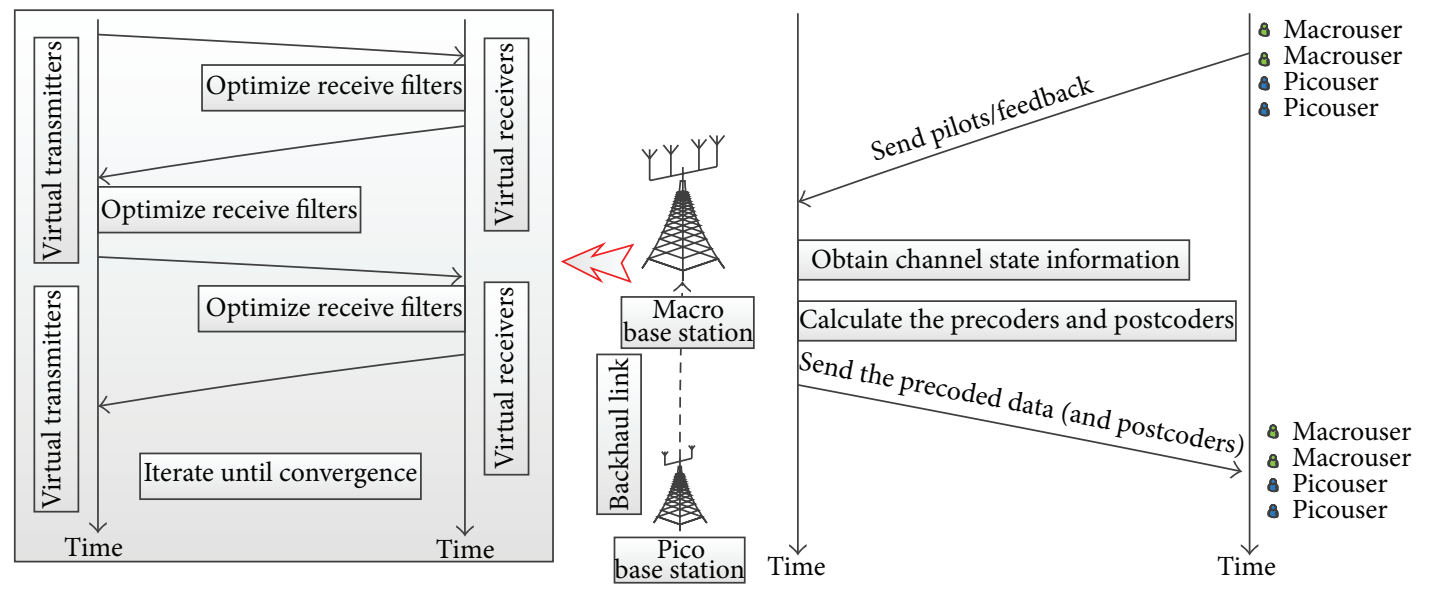

FIGURE 3: Macrointerference subspace reduction mechanism.

the algorithms in a generic framework to operate in Frequency Division Duplexing (FDD) or TDD modes as shown in Figure 3.

(2) Also, the receivers (UEs) have limited processing functionalities in practical systems. Henceforth, they cannot cope with algorithms that need high computational complexity. This can impose limitations to the complexity of the algorithms. Thus we need to design algorithms with low complexity and simple computation. In our scenario we assume all the computations are done within the macro BS which is equipped with powerful processing capabilities, thus relaxing this complexity limitations as shown in Figure 3.

(3) Moreover, for scalability issues, algorithms with lower complexity are always favoured, in order to extend the algorithms to large scale problems.

Here, our complexity criterion is the number of complex multiplications. The computational complexity for BDIA, BDIA uncord., and BDIA MMSE JTR algorithms is analyzed considering the $\mathrm{BD}$ as a baseline for all of them and thus not taken into account. The main computations in one iteration are listed as follows.

(1) The computation of the coordinated interference for all the three algorithms in (8), (12), and (15): the complexity is $K^{2}\left(s N_{t} N_{r}+s N_{r}^{2}\right)$, where $k$ is the number of UEs, $s$ is the number of streams per UE, and $N_{t}$ and $N_{r}$ represent the number of transmit and receive antennas, respectively.

(2) The eigenvalue decomposition carried out by the three algorithms in (8), (12), and (16): the complexity is $9 K\left(N_{r}^{3}\right)$.

(3) The computation of the intracluster interference which is caused by other cells within the same cluster in both BDIA uncord. and BDIA MMSE JTR algorithms shown in (13): the complexity is $K^{2}\left(s N_{t} N_{r}+\right.$ $s N_{r}^{2}$.
(4) The computation of the intercluster interference from the surrounding tiers in both BDIA uncord. and BDIA MMSE JTR algorithms shown in (14): the complexity is $K^{2}\left(s N_{t} N_{r}+s N_{r}^{2}\right)$.

(5) The computation of the objective function for all the three algorithms in (7) and (20): the complexity is $k(k-1) s^{2}\left(\min \left(N_{t}, N_{r}\right)+1\right)$ given that the interference terms complexity are already accounted previously.

(6) The computation of the matrix inversion in BDIA MMSE JTR algorithm shown in (18), (19), and (21): the complexity for each is $K\left(N_{r}-s\right)^{3}$.

The computational complexity comparison between BDIA, BDIA uncord., and BDIA MMSE JTR is summarized in Table 1. It is clear that BDIA MMSE JTR has the highest computation complexity per iteration followed by BDIA uncord. Finally BDIA has the lowest computational complexity per iteration.

\section{Numerical Results}

In order to evaluate the proposed algorithms, we simulated a realistic ultradense HetNet scenario, where the network is overloaded with a lot of UEs that need to be served simultaneously in the same time-frequency resource with high data rates. We carried out Monte-Carlo simulations with 500 runs. Each run is an independent (with uniformly distributed dropped users) channel realization.

Here, we consider a coordinated cluster to consist of one macrosector and the underlying small cells which are deployed randomly within the coverage area of the macrosector. The macrosector is the main entity in the cluster, while the small cells are deployed on demand when the number of UEs in the cluster increases. The small cell BS is equipped with 2 transmit antennas, while the macro BS is equipped with 8 transmit antennas. Throughout our simulation scenarios each small cell serves only 1 UE per RB, while the macro BS can serve UEs less than or equal to the number of its transmit antennas. A summary for the simulation parameters is shown in Table 2. 
TABLE 1: Comparison of computational complexity.

\begin{tabular}{lccc}
\hline Operation & BDIA & BDIA uncord. & BDIA MMSE JTR \\
\hline Coordinated interference $\left(K^{2}\left(s N_{t} N_{r}+s N_{r}^{2}\right)\right)$ & $\checkmark$ & $\checkmark$ & $\checkmark$ \\
Eigenvalue decomposition $\left(9 K\left(N_{r}^{3}\right)\right)$ & $\checkmark$ & $\checkmark$ & $\checkmark$ \\
Intracluster interference $\left(K^{2}\left(s N_{t} N_{r}+s N_{r}^{2}\right)\right)$ & & $\checkmark$ & $\checkmark$ \\
Intercluster interference $\left(K^{2}\left(s N_{t} N_{r}+s N_{r}^{2}\right)\right)$ & & $\checkmark$ & $\checkmark$ \\
Objective function $\left(k(k-1) s^{2}\left(\min \left(N_{t}, N_{r}\right)+1\right)\right)$ & $\checkmark$ & $\checkmark$ & $\checkmark$ \\
Matrix inversion $\left(K\left(N_{r}-s\right)^{3}\right)$ & & & $\checkmark$ \\
\hline
\end{tabular}

TABLE 2: Simulation parameter.

\begin{tabular}{ll}
\hline Parameter & Value \\
\hline Channel model & QUADRIGA [19] \\
Scenario & $\begin{array}{l}\text { Macro BS: urban macro (C2) } \\
\text { Small cell: urban mirco (B1) }\end{array}$ \\
Propagation & Non-line-of-sight \\
Large-scale fading & Geo-correlated parameters maps \\
Center frequency $f_{c}$ & $2.6 \mathrm{GHz}$ \\
Simulation type & Monte Carlo (500 runs) \\
Traffic model & Full buffer \\
Signal bandwidth & $180 \mathrm{kHz}$ per RB, 100 RBs \\
Intersite distance & $500 \mathrm{~m}$ \\
(macro) & 19 having 3 sectors each \\
\hline Number of macro BSs & $(1-10)$ per macrosector \\
Number of small cells & Macro: 4,$8 ; \lambda / 2 ;$ small cell: $2 ; \lambda / 2$ \\
$N_{t}$; spacing & Macro: 49 dBm; small cell: $26 \mathrm{dBm}$ \\
Transmit power & Macro: 32 m; small cell: $5 \mathrm{~m}$ \\
BS height & $75 \mathrm{~m}$ \\
Min. distance between \\
macrocell and small cell \\
Min. distance between
\end{tabular}

In Figure 4 we introduce two main simulation environments, which are the homogeneous environment, where only macro UEs are served and no small cells are deployed, and the ultradense heterogeneous one, where the macro sector is overloaded with small cells. In Figure 5 we compare the cluster sum spectral efficiency for 3 different scenarios. The first scenario is the homogeneous one; the second scenario is the ultradense heterogeneous one where the macro BS applies only $\mathrm{BD}$ algorithm and the cluster UEs apply the MMSE linear equalizer. Thus, this scenario is referred to as ultradense uncoordinated scenario since no coordination occurs between the macro and small cell BSs. The third scenario is the coordinated ultradense one, where the macro
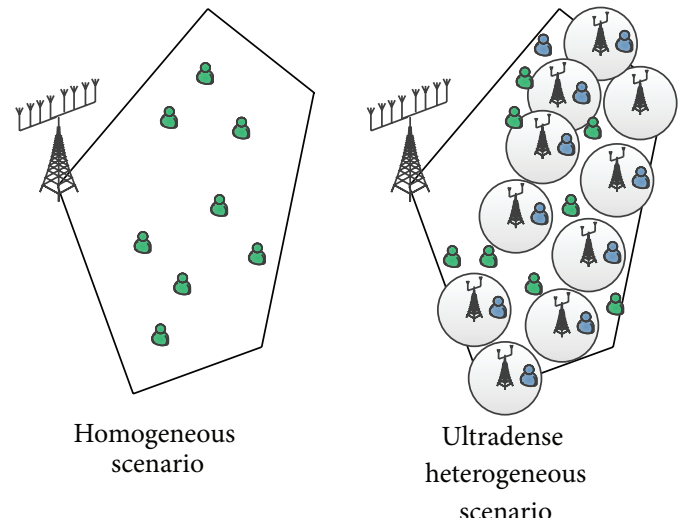

$\begin{array}{ll}0 \text { Macrosector } & \text { (1) Picocell } \\ 8 \text { Macrouser } & \text { \& Picouser }\end{array}$

FIGURE 4: Homogeneous and ultradense heterogeneous network deployments.

BS applies the BDIA MMSE JTR algorithm; thus coordination occurs between the macro and small cell BSs within the same cluster in this case.

As we can see in Figure 5 that the HetNet deployment always achieves higher sum spectral efficiency than the homogeneous one even when no coordination takes place between the macro and small cell BSs. Also, we can observe that coordinated ultradense scenario achieves higher spectral efficiency over the uncoordinated ultradense one only when enough free spatial dimensions are available at the macro BS to align the macrointerference towards the small cells. Here we can see that the coordinated beamforming achieves higher spectral efficiency than the uncoordinated one in ultradense deployment, until the case where 6 UEs are served per macro BS and two spatial dimensions are available at the macro BS for aligning the interference. However, once we move to the case where 7 UEs are served per macro BS and only 1 spatial dimension is free for interference alignment the sum spectral efficiency drops below the uncoordinated case.

In Figure 6, we consider the case where the macro BS has enough free spatial dimensions for aligning the interference. Here the macro BS is serving only 2 UEs, thus having 6 free spatial dimensions, while small cells are deployed from 1 to 10 and each small cell is serving 1 UE. We observe that even when the macro BS DoF are exceeded the BDIA achieves higher spectral efficiency than applying only BD algorithm at the macro BS. Moreover, the BDIA MMSE JTR which is 


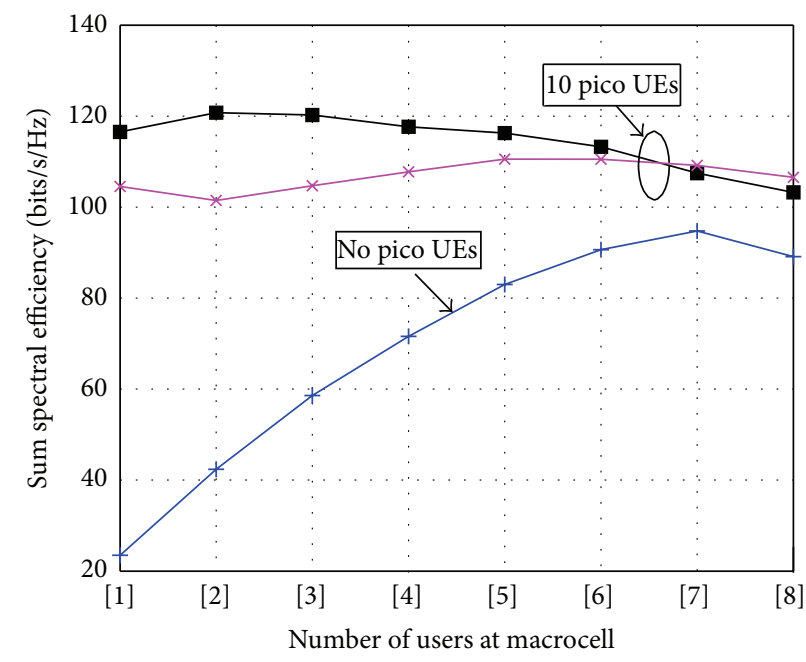

- BD MMSE Rx. (only macro UEs)

$\rightarrow$ BDIA MMSE JTR (coordinated)

* BD MMSE Rx. (uncoordinated and macro and pico UEs)

FIGURE 5: Comparing the sum spectral efficiency for homogeneous, uncoordinated ultradense and coordinated ultradense scenarios, with 8 transmitting antennas at the macro BS.

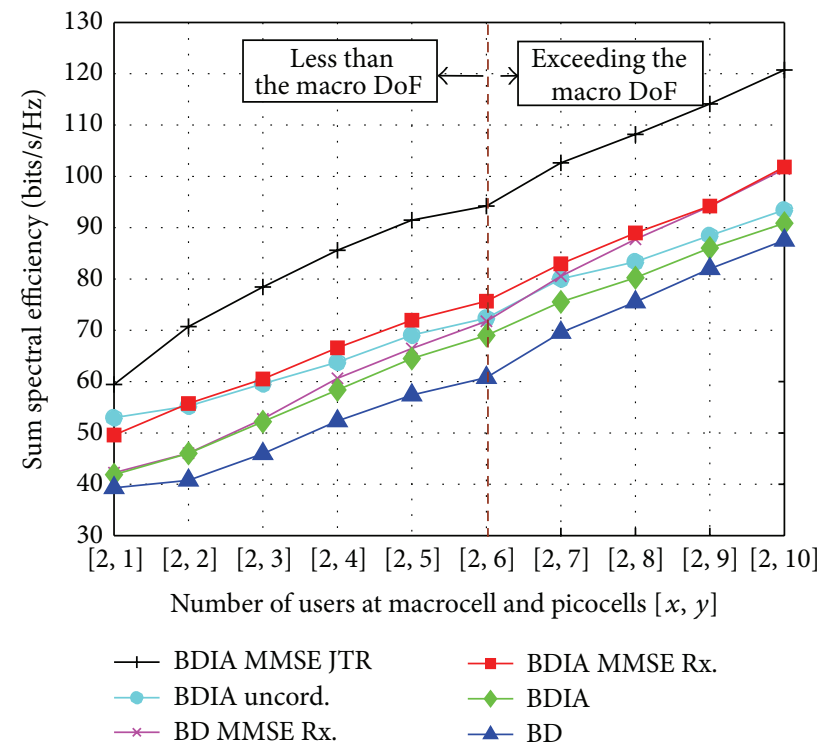

FIGURE 6: Evaluating the cluster sum spectral efficiency for serving 2 macro UEs and deploying small cells from 1 to 10 within the cluster for the introduced algorithms.

referred to as the coordinated beamforming case achieves the highest spectral efficiency with a gain of $20 \mathrm{bits} / \mathrm{s} / \mathrm{Hz}$ over the case when the macro BS applies only BD while each UE within the cluster applies MMSE equalizer, referred to as the uncoordinated beamforming case.

In order to inspect the results in Figure 6 in more detail we show the coordinated and uncoordinated interference power received by the small cell connected UEs in Figures 7 and 8, respectively. In Figure 7 it is shown that the BDIA algorithm can perfectly align the macrointerference towards

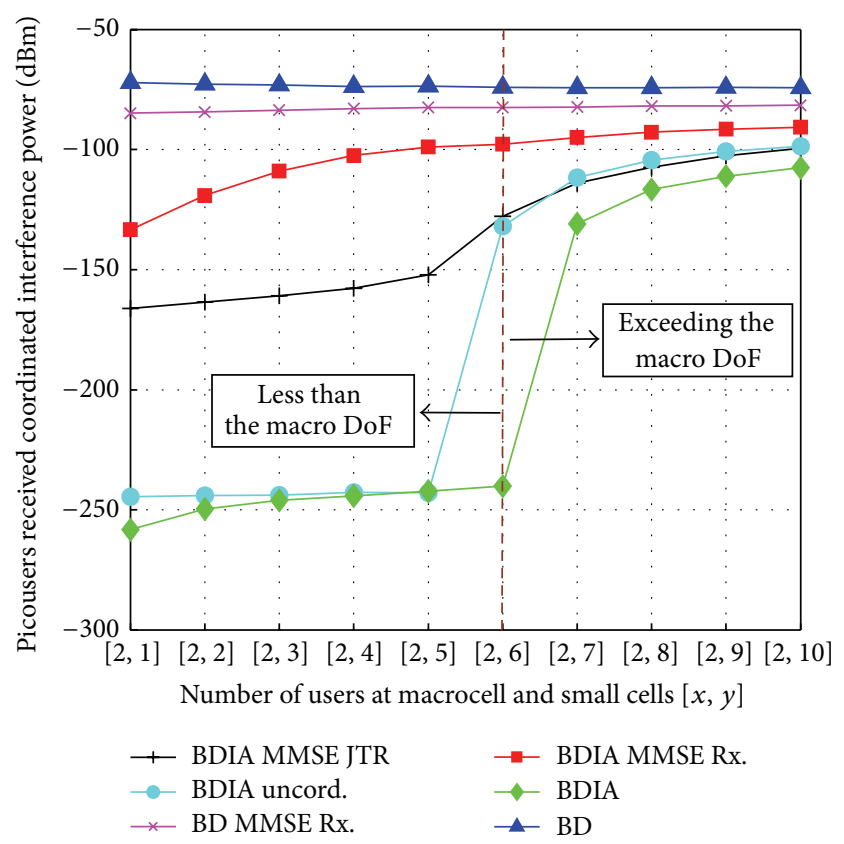

FIGURE 7: Evaluating the small cell connected UEs received coordinated interference power for serving 2 macro UEs and deploying small cells from 1 to 10 within the cluster.

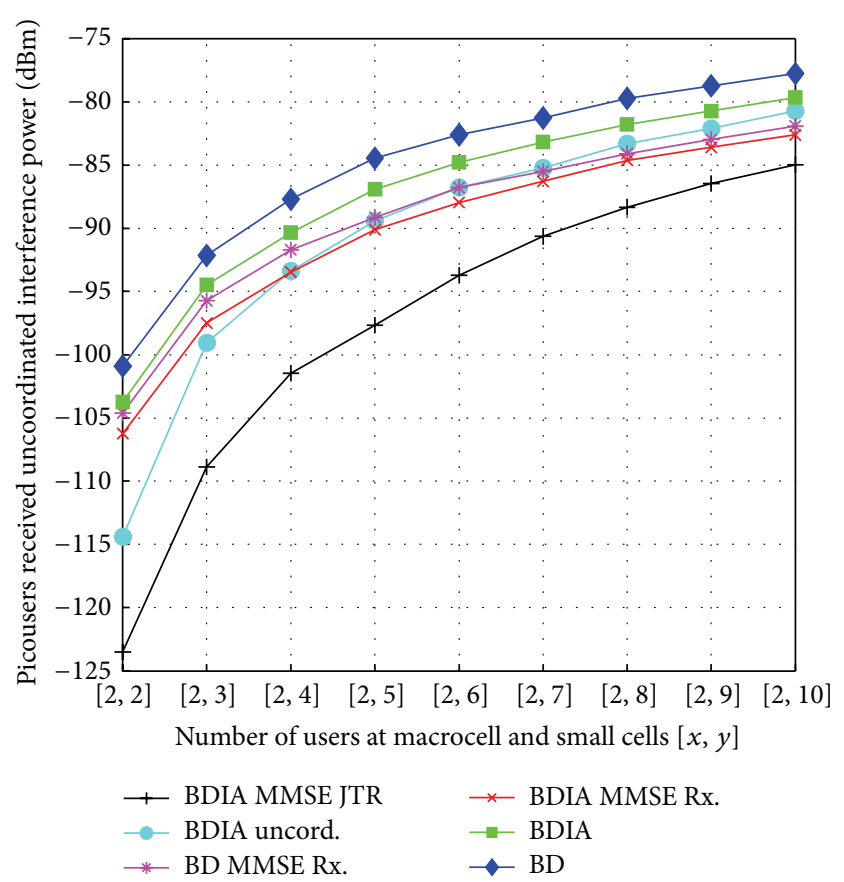

FIGURE 8: Evaluating the small cell connected UEs received uncoordinated interference power for serving 2 macro UEs and deploying small cells from 1 to 10 within the cluster.

the small cell connected UEs as long as the number of UEs within the cluster is less than or equal to the DoF available at the macro BS. When the number of the cluster UEs exceeds the macro DoF, the BDIA can no longer align the macro interference perfectly towards the small cell connected UEs. However, it can still partially align the macrointerference, thus applying BDIA at the macro BS achieves the lowest 


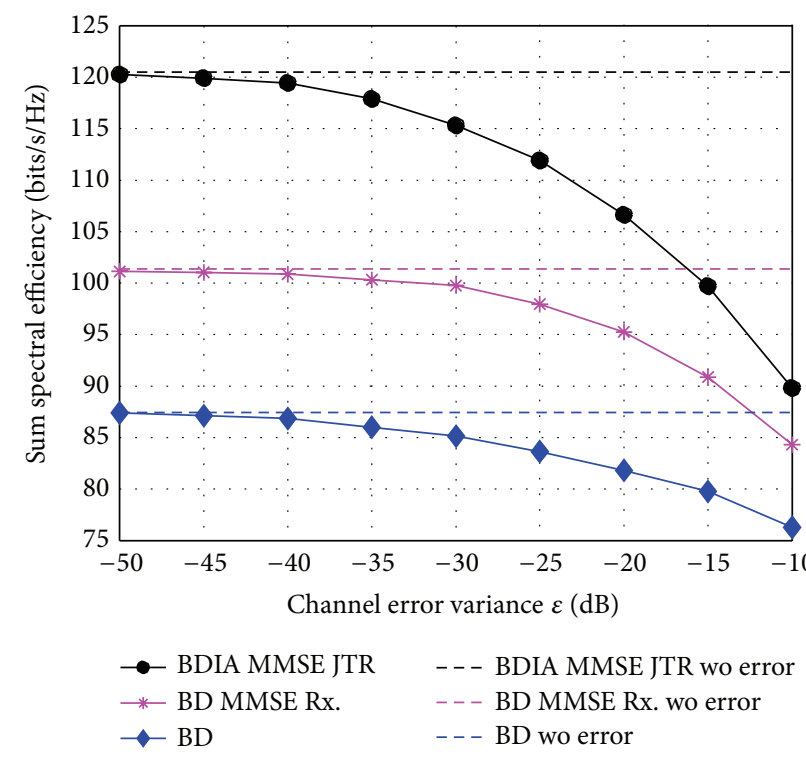

FIGURE 9: Evaluating the cluster sum spectral efficiency for serving 2 macro UEs and deploying 10 small cells within the cluster, in coordinated and uncoordinated scenarios (with and without perfect CSI).

received interference power at the small cell connected UEs side compared to all the other introduced algorithms, even when the number of UEs within the cluster exceeds the macro BS DoF. Moving to Figure 8 we can observe that applying the BDIA MMSE JTR algorithm at the macro BS achieves the lowest received uncoordinated interference power at the small cell connected UEs side compared to all the other introduced algorithms

In order to evaluate our framework in more realistic environment, we introduce the results for having imperfect CSI and evaluate the sensitivity of the introduced algorithms towards the channel error. In Figure 9 we observe that increasing the channel error variance $\epsilon$ from -50 to $-10 \mathrm{~dB}$ causes a drop in the sum spectral efficiency of $30 \mathrm{bits} / \mathrm{s} / \mathrm{Hz}$ in case of applying the BDIA MMSE JTR algorithm. However, it causes a drop of $16 \mathrm{bits} / \mathrm{s} / \mathrm{Hz}$ in case of applying the $\mathrm{BD}$ algorithm at the macro BS side while utilizing the MMSE linear equalizer at the receiver side. While, in case of applying only BD algorithm at the macro BS side, a drop of $12 \mathrm{bits} / \mathrm{s} / \mathrm{Hz}$ occurs.

Figure 10 shows the normalized degradation in sum spectral efficiency due to increasing the channel error variance. It is clear that the BDIA MMSE JTR is the most sensitive algorithm to channel error such that the sum spectral efficiency drops by $25 \%$, followed by the all other algorithms that utilize the IA concept (BDIA, BDIA uncord., and BDIA MMSE Rx.) with a degradation of about $21 \%$ followed by the BD with MMSE equalizer with a degradation of $17 \%$ and finally the BD algorithm with a degradation of $13 \%$.

Finally, one more step to make the simulation environment more realistic is introducing the MC scenario as shown in Figure 1, where the coordinated cluster is now deployed within two tiers of active macro BSs that are causing severe interference towards the coordinated cluster. In Figure 11 we compare the cluster sum spectral efficiency in the Single

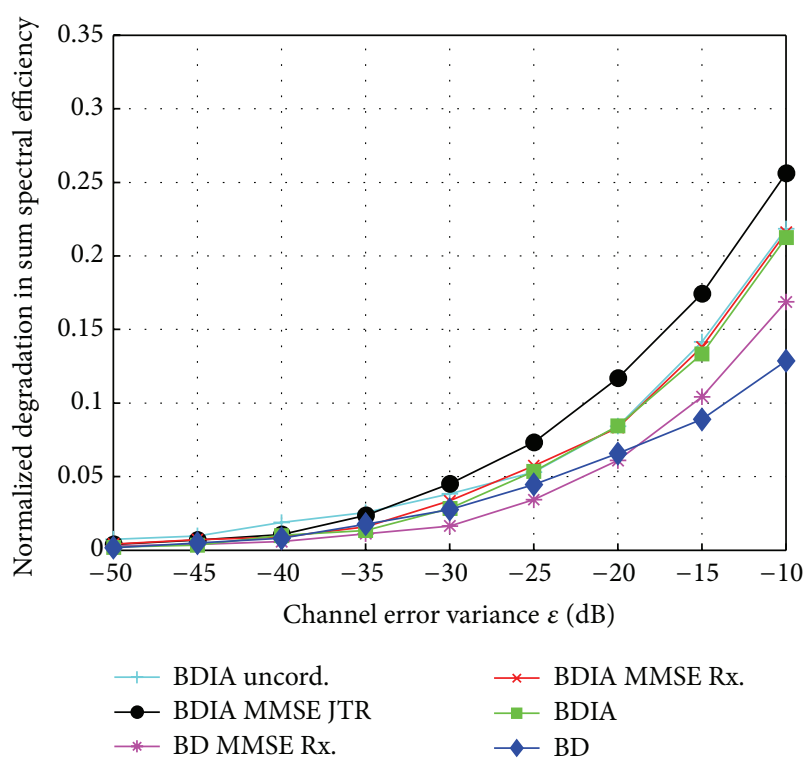

FIGURE 10: Evaluating the normalized degradation in cluster sum spectral efficiency for serving 2 macro UEs and deploying 10 small cells within the cluster with imperfect CSI.

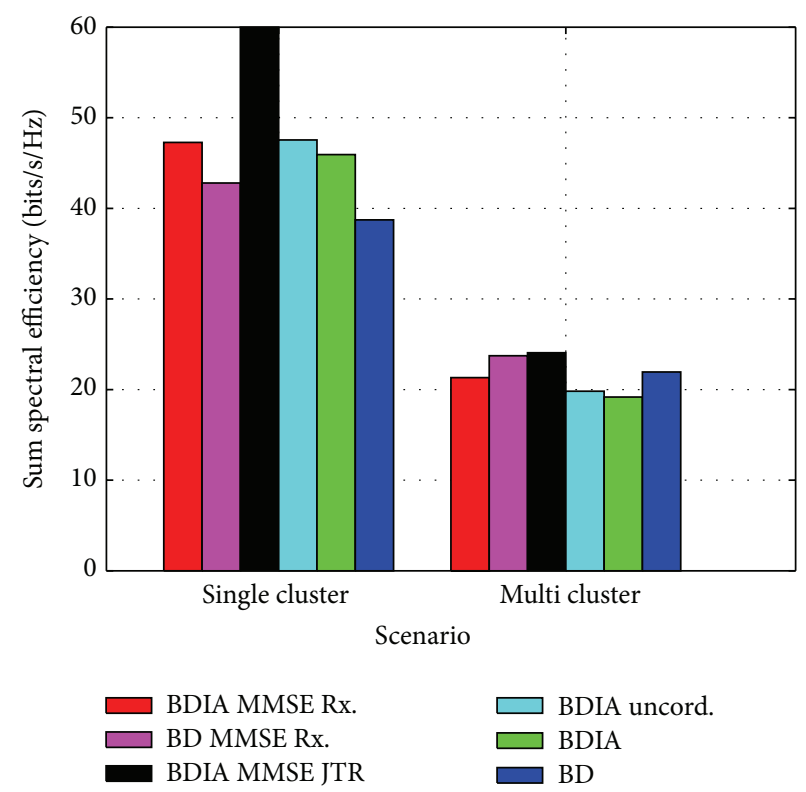

FIGURE 11: Cluster sum spectral efficiency comparison between the single and multicluster scenarios for serving 2 macro UEs and deploying 2 small cells within the cluster, with the macro BS employed with 4 transmitting antennas.

Cluster (SC) scenario with the MC one. In this deployment the macro BS is deployed with 4 transmit antennas and serving 2 UEs, while 2 small cells are deployed within the coverage of the macrosector; each is deployed with 2 transmit antennas and serving $1 \mathrm{UE}$. It is shown in Figure 11 that moving from the SC scenario to the MC one causes a severe degradation in the cluster sum spectral efficiency for all the introduced algorithms, specially for the BDIA MMSE JTR algorithm which suffers from a degradation of $35 \mathrm{bits} / \mathrm{s} / \mathrm{Hz}$. 


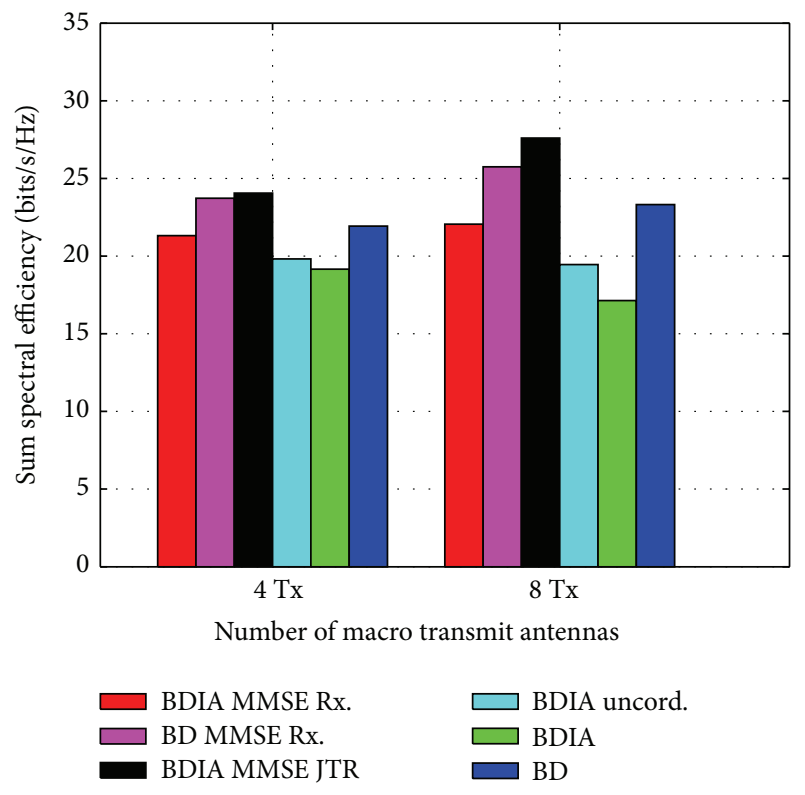

FIGURE 12: The effect of increasing the number of macro transmit antennas on the cluster sum spectral efficiency for serving 2 macro UEs and deploying 2 small cells within the cluster.

This degradation makes the BDIA MMSE JTR which is referred to as coordinated beamforming give a higher sum spectral efficiency of only $0.5 \mathrm{bits} / \mathrm{s} / \mathrm{Hz}$ over the $\mathrm{BD}$ with MMSE equalizer which is referred to as uncoordinated beamforming, in the MC case.

In order to exploit the gain from using the coordinated beamforming we increase the number of macro transmit antennas to 8 . The gain from increasing the macrospatial dimensions is shown in Figure 12. We can observe that increasing the number of macro transmit antennas results in a gain for all the introduced algorithms and specially for the coordinated beamforming scheme, such that the gain of the coordinated beamforming scheme over the uncoordinated one increased to $2.5 \mathrm{bits} / \mathrm{s} / \mathrm{Hz}$ instead of only $0.5 \mathrm{bits} / \mathrm{s} / \mathrm{Hz}$ in the case when the macro BS was equipped with only 4 transmit antennas. This result means that increasing the macrospatial dimensions allows us to yield higher gains in the cluster sum spectral efficiency when applying the coordinated beamforming scheme. Henceforth, moving to the massive MIMO regime is expected to achieve high gains for the introduced hierarchical coordinated beamforming schemes.

\section{Conclusion}

From the results shown in Section 5 we can conclude that the hierarchical precoding framework performs higher spectral efficiency than the uncoordinated beamforming given that a SC scenario is available, with enough free spatial dimensions at the macro BS for aligning the interference. This is valid even when the number of the UEs within the cluster exceeds the DoF available at the macro BS. We can also conclude that robust coordinated beamforming cannot be totally ensured with the introduced hierarchical precoding framework in ultradense HetNet scenario, due to the high sensitivity to imperfect CSI, even if a SC scenario is available with enough free spatial dimensions at the macro BS. Moreover, we can observe that a severe degradation happens in the sum spectral efficiency for the hierarchical framework in the MC scenario (when multiple sources of uncoordinated interference exist with high power and different directions). Thus, a solution for this problem is increasing the number of the available spatial dimensions at the macro BS. This was shown in Section 5, where increasing the number of macro transmit antennas from 4 to 8 achieved a high gain for the coordinated beamforming scheme.

Therefore as a future work, we suggest increasing the number of the spatial dimensions by either increasing the number of macro BS transmit antennas or increasing the number of the UEs receive antennas. Concerning the imperfect CSI scenario, we also suggest as a future work to introduce adaptive precoding technique enclosing all the proposed algorithms within the paper and even more algorithms that are more robust against channel errors. Henceforth, each algorithm can be employed based on a threshold for the accuracy of the CSI, with considering the trade-off between the spectral efficiency, the complexity, and the overhead of each algorithm.

\section{Competing Interests}

The authors declare that they have no competing interests.

\section{Acknowledgments}

Part of this work has been performed in the framework of the Horizon 2020 project Flexible Air iNTerfAce for Scalable service delivery wiThin wIreless Communication networks of the 5th Generation (FANTASTIC-5G) (ICT-671660), which is partly funded by the European Union. The authors would like to acknowledge the contributions of their colleagues in FANTASTIC-5G.

\section{References}

[1] V. Jungnickel, K. Manolakis, W. Zirwas et al., "The role of small cells, coordinated multipoint, and massive MIMO in 5G," IEEE Communications Magazine, vol. 52, no. 5, pp. 44-51, 2014.

[2] J. F. Monserrat, H. Droste, Ó. Bulakci et al., "Rethinking the mobile and wireless network architecture: the METIS research into 5G," in Proceedings of the European Conference on Networks and Communications (EuCNC '14), pp. 1-5, June 2014.

[3] N. Bhushan, J. Li, D. Malladi et al., "Network densification: the dominant theme for wireless evolution into 5G," IEEE Communications Magazine, vol. 52, no. 2, pp. 82-89, 2014.

[4] S. Yunas, M. Valkama, and J. Niemelä, "Spectral and energy efficiency of ultra-dense networks under different deployment strategies," IEEE Communications Magazine, vol. 53, no. 1, pp. 90-100, 2015.

[5] E. G. Larsson, O. Edfors, F. Tufvesson, and T. L. Marzetta, "Massive MIMO for next generation wireless systems," IEEE Communications Magazine, vol. 52, no. 2, pp. 186-195, 2014.

[6] T. L. Marzetta, "Massive MIMO: an introduction," Bell Labs Technical Journal, vol. 20, pp. 11-12, 2015. 
[7] T. L. Marzetta, "Noncooperative cellular wireless with unlimited numbers of base station antennas," IEEE Transactions on Wireless Communications, vol. 9, no. 11, pp. 3590-3600, 2010.

[8] 3GPP, "Physical channels and modulation," 3rd Generation Partnership Project Standard 36 211, 3GPP, 2016.

[9] V. Cadambe and S. Jafar, "Interference alignment and spatial degrees of freedom for the $\mathrm{k}$ user interference channel," in Proceedings of the in IEEE International Conference on Communications (ICC '08), pp. 971-975, May 2008.

[10] M. Maddah-Ali, A. Motahari, and A. Khandani, "Communication over mimo x channels: interference alignment, decomposition, and performance analysis," IEEE Transactions on Information Theory, vol. 54, no. 8, pp. 3457-3470, 2008.

[11] S. W. Peters and R. W. Heath Jr., "Interference alignment via alternating minimization," in Proceedings of the IEEE International Conference on Acoustics, Speech, and Signal Processing (ICASSP '09), pp. 2445-2448, IEEE, Taipei, Taiwan, April 2009.

[12] Q. H. Spencer, A. L. Swindlehurst, and M. Haardt, "Zero-forcing methods for downlink spatial multiplexing in multiuser MIMO channels," IEEE Transactions on Signal Processing, vol. 52, no. 2, pp. 461-471, 2004.

[13] S. W. Peters and R. W. Heath Jr., "Cooperative algorithms for MIMO interference channels," IEEE Transactions on Vehicular Technology, vol. 60, no. 1, pp. 206-218, 2011.

[14] J. Dommel, P.-P. Knust, L. Thiele, and T. Haustein, "Massive MIMO for interference management in heterogeneous networks," in Proceedings of the IEEE 8th Sensor Array and Multichannel Signal Processing Workshop (SAM '14), pp. 289292, A Coruna, Spain, June 2014.

[15] M. Kurras, M. Shehata, K. Hassan, and L. Thiele, "Spatial interference management with hierarchical precoding in ultradense heterogeneous networks," in Proceedings of the IEEE 11th International Conference on Wireless and Mobile Computing, Networking and Communications (WiMob '15), pp. 520-526, IEEE, Abu Dhabi, United Arab Emirates, October 2015.

[16] L. Thiele and M. Kurras, "Hierarchical precoding for ultradense heterogeneous networks," in Proceedings of the 48th Asilomar Conference on Signals, Systems and Computers (ACSSC '15), pp. 1286-1290, November 2014.

[17] C. Sun, Y. Yang, and Y. Yuan, "Low complexity interference alignment algorithms for desired signal power maximization problem of MIMO channels," Eurasip Journal on Advances in Signal Processing, vol. 2012, article 137, 2012.

[18] H. G. Ghauch and C. B. Papadias, "Interference alignment: a onesided approach," in Proceedings of the IEEE Global Telecommunications Conference (GLOBECOM '11), pp. 1-5, Houston, Tex, USA, December 2011.

[19] S. Jaeckel, L. Raschkowski, K. Borner, and L. Thiele, "QuaDRiGa: a 3-D multi-cell channel model with time evolution for enabling virtual field trials," IEEE Transactions on Antennas and Propagation, vol. 62, no. 6, pp. 3242-3256, 2014. 


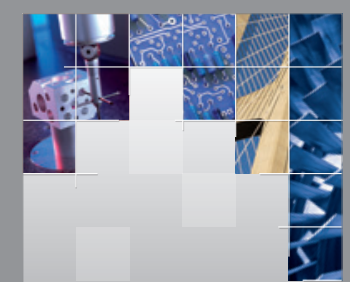

\section{Enfincering}
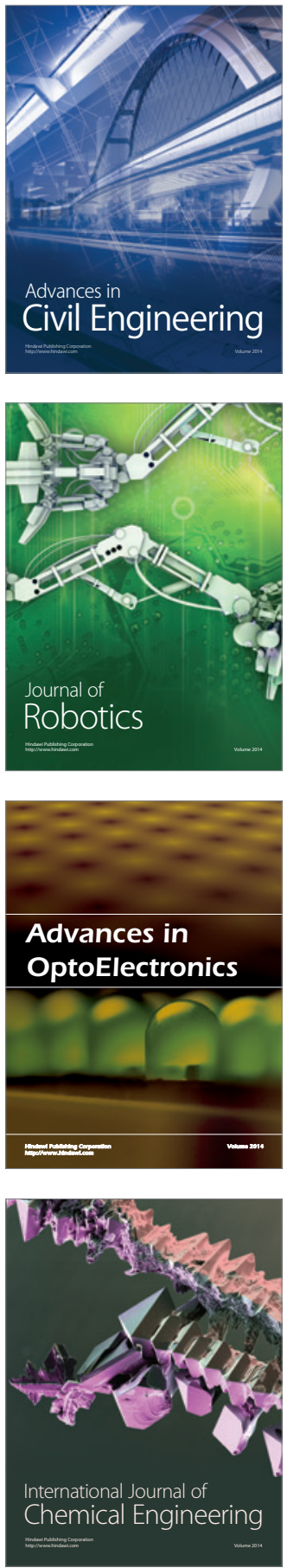

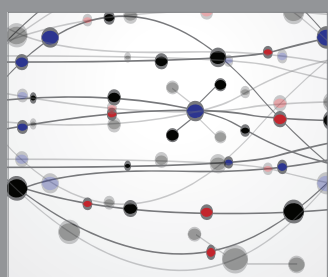

The Scientific World Journal

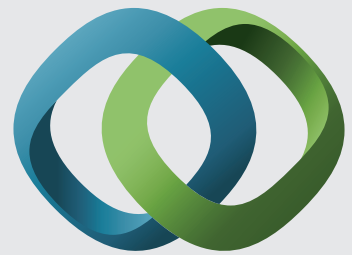

\section{Hindawi}

Submit your manuscripts at

http://www.hindawi.com
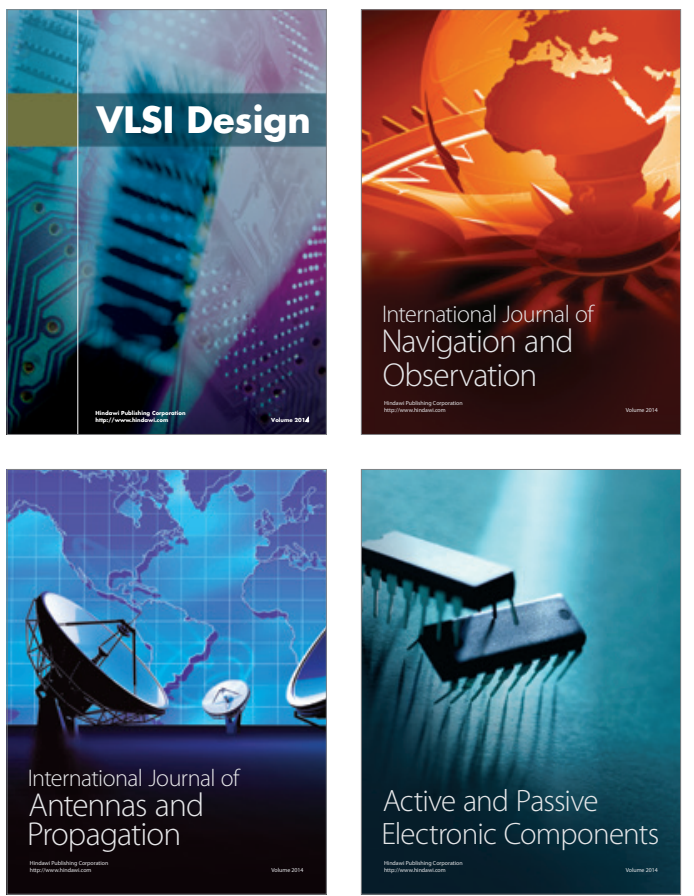
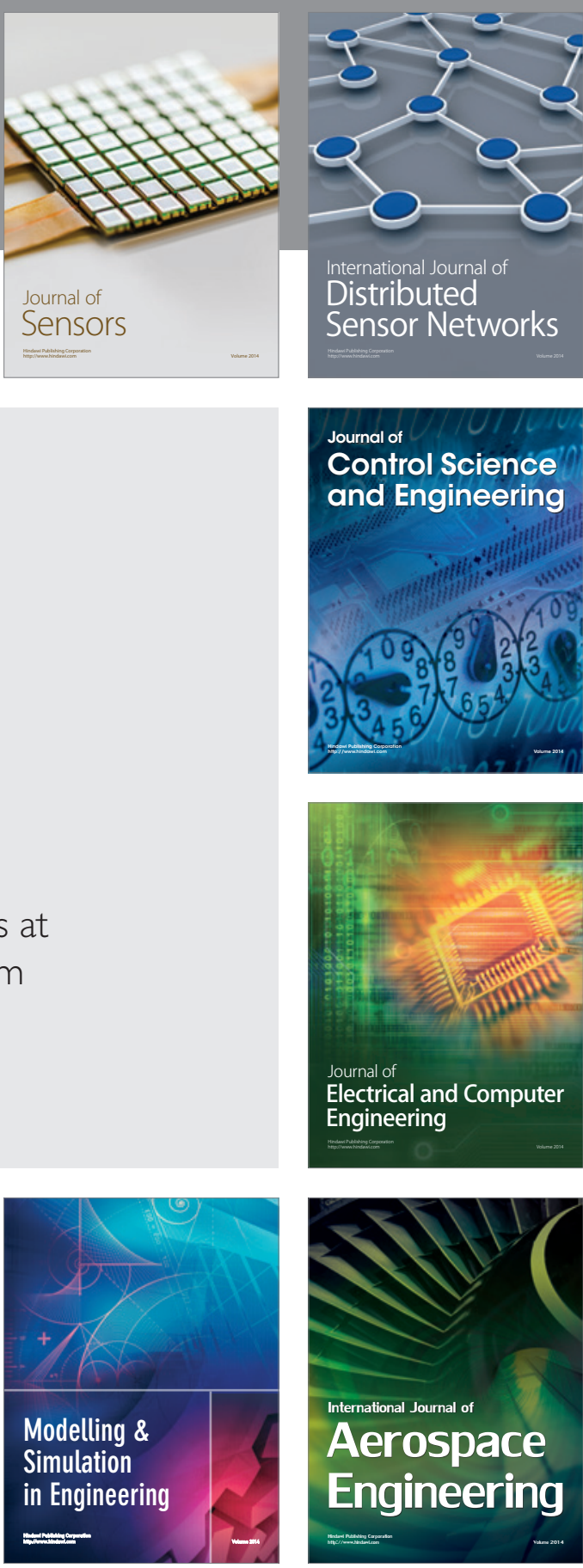

International Journal of

Distributed

Sensor Networks

Journal of

Control Science

and Engineering
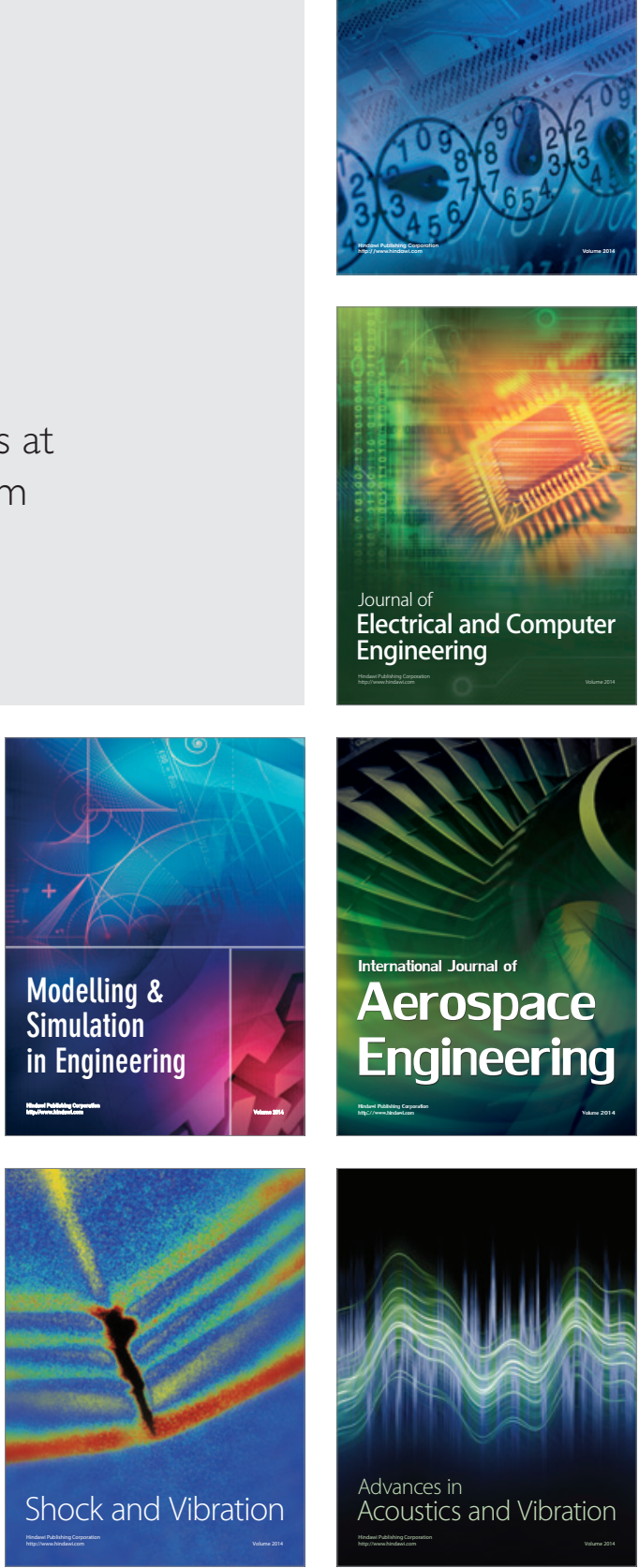